\title{
Intakes and status of riboflavin in a representative sample of Irish adults aged 18-90 years screened for MTHFR C677T polymorphism
}

\author{
Emma O’Sullivan $^{1}$, Laura Kehoe ${ }^{1}$, Janette Walton ${ }^{2,1}$, Helene McNulty ${ }^{3}$, Mary Ward ${ }^{3}$, \\ Liadhan McAnena $^{3}$, Sinead M. Hopkins ${ }^{4}$, Breige A. McNulty ${ }^{4}$, Anne P. Nugent ${ }^{4,5}$ and \\ Albert Flynn ${ }^{1}$ \\ ${ }^{1}$ School of Food and Nutritional Sciences, University College Cork, Cork, Ireland, \\ ${ }^{2}$ Department of Biological Sciences, Cork, Ireland, \\ ${ }^{3}$ Nutrition Innovation Centre for Food and Health, Ulster University, Coleraine, United Kingdom, \\ ${ }^{4} U C D$ Institute of Food and Health, University College Dublin, Dublin, Ireland and \\ ${ }^{5}$ Institute for Global Food Security, School of Biological Sciences, Queens University Belfast, Belfast, United Kingdom
}

\begin{abstract}
Meta-analyses of epidemiological data report that adults who carry a common polymorphism, the $M T H F R 677 \mathrm{C} \rightarrow \mathrm{T}$, in the gene encoding the folate-metabolising enzyme methylenetetrahydrofolate reductase (MTHFR) have a $40 \%$ increased risk of CVD and an $87 \%$ increased risk of hypertension. Riboflavin (vitamin B2), in its co-enzymatic form flavin adenine nucleotide (FAD), is required as a co-factor by MTHFR and previous trials in hypertensive patients have shown a blood pressure lowering response to riboflavin supplementation that is specific to individuals homozygous for this polymorphism (TT genotype). Low folate status is commonly reported in adults with the TT genotype however the effect of this genetic variant on riboflavin status has not previously been investigated. The aim of this study, therefore, was to investigate dietary intake and biomarker status of riboflavin by MTHFR genotype in Irish adults using data from the National Adult Nutrition Survey (2008-2010) (www.iuna.net).

A 4-day semi-weighed food record was used to collect food and beverage intake data from a representative sample of 1500 Irish adults (18-90 years). Dietary intake data were analysed using WISP ${ }^{\odot}$ based on UK food composition tables (modified to include recipes of composite dishes, nutritional supplements, fortified foods and generic Irish foods that were commonly consumed). Usual intakes were calculated via the NCI-method using SAS ${ }^{\odot}$ Enterprise Guide. Blood samples $(n=1126)$ were collected by venepuncture by a trained professional and were processed and analysed using standard operating procedures. Biomarker status of riboflavin was determined by erthyrocyte gluthathione reductase activation coefficient (EGRac), a functional assay that measures the activity of the enzyme glutathione reductase before and after in vitro activation with its prosthetic group FAD; a lower value indicates better status.

It was found that $12 \%$ of the population had the TT genotype. As expected, there was no significant difference in riboflavin intake across the genotype (CC, CT or TT) groups. Similarly, no significant genotype differences in riboflavin status (EGRac) were observed (1.36 vs 1.37 vs 1.38 respectively). Overall, $61 \%$ of the total population had EGRac values $>1.3$, indicative of low/deficient status with no significant difference observed between the genotype groups $(60 \%, 61 \%$ and $61 \%$, respectively).

These data suggest that riboflavin status is not influenced by the C677T polymorphism in MTHFR in this cohort of nationally representative Irish adults. Further research is needed to see the impact of riboflavin status on blood pressure across the genotype groups in this nationally representative cohort of Irish adults.
\end{abstract}

\section{Conflict of Interest}

There is no conflict of interest. 